\title{
Documentos en contexto: las nuevas normas de descripción archivística y su aplicación en la administración digital
}

\section{Javier Requejo Zalama}

Ministerio de Educación, Cultura y Deporte SUbDIRECCIÓN GENERAL DE ARCHIVOS ESTATALES

\section{Resumen}

A partir de una reflexión inicial sobre el origen del proceso de normalización de la descripción archivística en España, se realiza una somera presentación de las principales normas de descripción desarrolladas a lo largo de los últimos 20 años. Se analiza la compleja relación entre ellas, especialmente en los actuales entornos de administración electrónica, donde prácticamente ninguna de las normas vigentes hasta el año 2015 era capaz de ofrecer una solución integral para todo el proceso descriptivo.

Esta reflexión se acompaña de una presentación de las últimas normas sobre la materia, especialmente de los modelos conceptuales de descripción archivística: NEDA-MC, concebido como norma española de descripción archivística y aprobado en 2017, y RiC-CM, de carácter internacional y todavía en fase de borrador. Ambas buscan solventar los problemas existentes puesto que identifican las entidades básicas a describir y las posibles relaciones entre ellas, concebidos ambos elementos como generadores del contexto informativo. Con ello, se erigen en unas nuevas normas para diseñar los sistemas de descripción, logrando la compatibilidad entre los diversos desarrollos normativos. 
Palabras clave: Descripción; Normalización; Norma Española de Descripción Archivística; Sistemas de descripción; Contexto documental

Cita recomendada: Requejo Zalama, J. (2018). Documentos en contexto: las nuevas normas de descripción archivística y su aplicación en la administración digital. En El archivo electrónico en la administración digital: 23 Jornadas de Archivos Universitarios, 21-23 de junio de 2017, A Coruña (pp. 27-41).

DOI capítulo: https://doi.org/10.17979/spudc.9788497496803.027

DOI libro: https://doi.org/10.17979/spudc.9788497496803

La descripción es un proceso de larga tradición en el ámbito de la gestión documental, prácticamente inherente a la propia historia de la gestión de los documentos. Ahora bien, la normalización de dicho proceso, entendida como la adopción de reglas comunes para llevar a cabo el proceso descriptivo, tiene un origen bastante más reciente. Y precisamente los avances en materia de descripción archivística han evolucionado tan rápido en las últimas décadas, que las normas para describir no eran capaces de dar una respuesta eficaz a las necesidades que planteaban esos nuevos entornos evolucionados.

\section{Análisis preliminar de la realidad normativa}

Para poder comprender el enorme avance que la descripción archivística ha experimentado en los últimos años, es preciso identificar el punto de origen del proceso normalizador. En el caso español, ese momento lo podemos situar en la década de los años 90 del pasado siglo XX, cuando 
la descripción en archivos venía manteniendo un modelo tradicional de descripción, asentado en una serie de prácticas que habían resultado de suma utilidad durante mucho tiempo. Incluso en determinados sectores se habían llegado a considerar normas de facto para describir como, por ejemplo, la identificación de las guías, inventarios y catálogos como instrumentos básicos para describir en archivos.

Es en esta década de los años 90 cuando la normalización se empieza a hacer efectiva a nivel global, con el primer gran proyecto de creación de una norma para describir en archivos a nivel internacional. Ese proyecto, si bien no llegó a cristalizar de forma efectiva hasta el año 2000 con la adopción de la segunda versión de $\operatorname{ISAD}(\mathrm{G})$, fue desarrollado durante la década anterior y llevó aparejado un impulso colectivo por adaptarse a la nueva situación.

Es en esa época cuando nace el espíritu normalizador en materia de descripción, resultando particularmente interesante analizar cómo ese proceso regulador surge en una etapa concreta de los archivos. También los archiveros y archiveras presentaban unas características definitorias muy específicas y ajustadas a esa época en concreto. Todos han sufrido una enorme actualización. Es el caso, por ejemplo, de la aparición de la administración electrónica, que ni siquiera era concebida como una posibilidad real en la década de los años 90 .

Esta situación inicial fue evolucionando en los años posteriores, momento en que se produce la consolidación de la descripción normalizada. En los quince primeros años del siglo XXI se produce el gran salto que abre un nuevo escenario para la gestión documental: la irrupción general de lo digital y, en particular, de la administración electrónica. Es precisamente con este salto cuando empiezan a plantearse las primeras cuestiones o dudas sobre las normas desarrolladas, pues estas no son capaces de 
ofrecer una solución a las nuevas coyunturas planteadas y se proponen soluciones específicas para darles respuesta ${ }^{4}$.

Además, el desarrollo de este nuevo entorno se apoyó en un cambio generacional, con la llegada al mundo de la gestión documental de un equipo de profesionales que tenían un mayor conocimiento sobre el uso de las tecnologías de información. Esto facilitó la aparición de ciertos estándares sectoriales, que pretendían resolver la carencia que la normalización inicial presentaba en esta nueva realidad. E incluso, en algunos de estos desarrollos sectoriales se puede llegar a atisbar lo que vendrá a ser el nuevo paradigma de la descripción archivística.

Lo cierto es que, en estos años de consolidación, la normalización de la descripción archivística eclosiona con la aparición, casi exponencial, de múltiples normas para describir, planteadas desde múltiples perspectivas:

- Según el objetivo de la norma, es posible clasificarlas de la siguiente manera:

- Estándares para identificar el contenido a describir (Tabla 1).

- Estándares para identificar la estructura de los datos (Tabla 2).

- Según el ámbito de aplicación de la norma es posible clasificarlas:

○ Estándares globales, de aplicación en cualquier entorno (cfr. Tabla 3).

- Estándares sectoriales, de aplicación limitada a determinados sectores territoriales, institucionales o administrativos (cfr. Tabla 4).

\footnotetext{
${ }^{4}$ Ninguna de las normas actuales adoptadas por el Consejo Internacional de Archivos (ISAD $(\mathrm{G})$, ISAAR(CPF), ISDF e ISDIAH) fueron concebidas para identificar campos o metadatos descriptivos, por lo que resultaban normas "inútiles" para diseñar sistemas de descripción. Hubo, por tanto, que plantearse requisitos funcionales específicos para crear aplicaciones informáticas de gestionar metadatos.
} 
Tabla 1. Normas de descripción de contenido

\begin{tabular}{lll} 
Objeto a describir & Estándares globales & Estándares sectoriales \\
\hline Documentos & ISAD(G) & MDM \\
& & NOGADA \\
& & NODAC \\
\multirow{2}{*}{ Agentes } & ISAAR(CPF) & ARANOR \\
& ISDIAH & \\
Funciones & ISDF &.-- \\
\hline
\end{tabular}

Tabla 2. Normas de descripción de estructura

\begin{tabular}{lll} 
Objeto a describir & Estándares globales & Estándares sectoriales \\
\hline Documentos & EAD & EMCAU \\
& & EDARA \\
& & VMGC \\
& & e-EMGDE \\
Agentes & EAC & e-EMGDE \\
& EAG & \\
Funciones &.-- & e-EMGDE \\
\hline
\end{tabular}

Tabla 3. Normas de descripción de aplicación global

\begin{tabular}{lll} 
Objeto a describir & $\begin{array}{l}\text { Estándares de } \\
\text { contenido }\end{array}$ & $\begin{array}{l}\text { Estándares de estructura de } \\
\text { datos }\end{array}$ \\
\hline Documentos & ISAD $(\mathrm{G})$ & EAD \\
Agentes & ISAAR $(\mathrm{CPF})$ & EAC \\
& ISDIAH & EAG \\
Funciones & ISDF &.-- \\
\hline
\end{tabular}


Tabla 4. Normas de descripción de aplicación sectorial

\begin{tabular}{lll} 
Objeto a describir & $\begin{array}{l}\text { Estándares de } \\
\text { contenido }\end{array}$ & $\begin{array}{l}\text { Estándares de estructura de } \\
\text { datos }\end{array}$ \\
\hline Documentos & MDM & EMCAU \\
& NOGADA & EDARA \\
& NODAC & VMGC \\
& & e-EMGDE \\
Agentes & ARANOR & e-EMGDE \\
Funciones &.-- & e-EMGDE \\
\hline
\end{tabular}

Con el paso de los años, esta situación derivó en un complejo escenario con múltiples normas para describir en archivos, desarrolladas por órganos de normalización muy diversos para cumplir cada una de ellas con finalidades muy específicas, cuyo cumplimiento, en ocasiones, resultaba incompatible con el de otras.

Esta realidad trajo como principal consecuencia el desencanto con la normalización tal y como se venía asumiendo desde sus primeros años. Se llegó incluso a descartar algunas de las normas de descripción básicas como elementos normativos, y se buscaron alternativas en ámbitos ajenos al mundo de la gestión documental donde, aparentemente, no existía un consenso regulador suficiente y en cuyas opciones no se prestaba especial atención a los principios básicos de la gestión documental.

Era necesario, por tanto, ofrecer una solución eficaz y segura ante esta peligrosa situación de desencanto, afrontando el reto de diseñar un nuevo escenario normativo que resolviera la situación, aglutinara todos los desarrollos existentes ofreciendo un mecanismo de coordinación entre ellos y evitará, en todo caso, convertirse en una norma más dentro de ese panorama superpoblado de reglas de descripción. 


\section{Replanteamiento del proceso: documentos en contexto y nuevas normas}

Para poder comprender mejor cómo obtener una solución eficaz a esta situación, debemos analizar la forma en que se aplican las normas de descripción actuales.

Para ello, si analizamos el proceso de descripción desde una perspectiva secuencial, entendido como un conjunto de actividades encaminadas a representar información sobre los documentos, lo que estamos haciendo es identificar una serie de elementos informativos que están presentes en los documentos y representarlos mediante sus correspondientes metadatos.

Visto así, en realidad se trata de un proceso sencillo y lineal en el que las normas de descripción actuales cumplen un papel regulador de los elementos informativos a describir, ya sea identificando el tipo de información o la estructura a la que esa información debe ajustarse. En función de la norma o normas que deseemos aplicar, los elementos informativos y su estructura serán distintos. Una vez planteado así, el proceso puede ejecutarse tantas veces como sea necesario, generando descripciones ajustadas a esas normas en las que, en última instancia, la selección de información se realiza individualmente en cada descripción.

Pero si analizamos ese mismo proceso, no ya desde una perspectiva basada en las actuaciones a realizar, sino desde la perspectiva del tipo de información que se representa al describir, la percepción del proceso descriptivo puede variar.

Cuando se identifica la información a describir, se puede llegar a una conclusión básica: no toda la información representada en la descripción de un documento corresponde a ese documento, sino también al contexto 
que lo rodea. Hay información que, estando vinculada al documento, desde una perspectiva teórica, en realidad corresponde a otros elementos (por ejemplo, nombres de instituciones, normas que lo regulan, personas implicadas, procedimientos aplicados, etc.). Lo más común es que ese tipo de información no se use de forma exclusiva en una única descripción, sino que puede ser empleada en múltiples descripciones al mismo tiempo. Por ello, puede ser tratada de forma independiente a las descripciones de los documentos, llegando incluso a disponer de repositorios específicos para la gestión de dichas descripciones. Por ejemplo, repositorios de procedimientos donde gestionar las descripciones de actividades, o directorios donde gestionar las descripciones de instituciones o personas implicadas en la gestión de los documentos.

Identificando estos diversos elementos como entidades diferenciadas de las descripciones de los propios documentos, se puede obtener una red de descripciones archivísticas que conecta las descripciones de los documentos, no sólo con relaciones entre ellas mismas, sino también a través de otras múltiples formas de relaciones entre instituciones, funciones, procedimientos, normas, etc.

De esta forma, el panorama donde se gestionan las descripciones se puede concebir como un sistema extensible ad infinitum en el que los documentos reflejan la misma información inicialmente requerida pero resultan accesibles de muchísimas formas no planteadas mediante un proceso descriptivo lineal. Todo ello permite el uso compartido de información, ahorrando costes de tratamiento y haciendo más eficiente la gestión de la información.

Esta nueva perspectiva de la descripción recibe la denominación de descripción de documentos en contexto, en la que ese contexto se configura como la información representada mediante el uso de 
relaciones entre múltiples entidades; de forma que cuantas más relaciones puedan desplegarse, de mejor calidad resultará la información por ser más accesible (Figura 1).

Este planteamiento de la descripción de los documentos en su contexto precisa de una nueva concepción de la descripción, entendiendo que es preciso identificar la forma en que los sistemas de descripción pueden gestionar las entidades a describir (documentos, instituciones, procedimientos, normas, etc.) y las posibles relaciones entre ellas. Esto implica una labor de estandarización del proceso de descripción, no ya en el momento de identificar la información que se va a representar, sino en una etapa anterior, concretamente aquella en la que se establecen los requisitos que debe cumplir el sistema que se va utilizar para describir. Es decir, qué entidades archivísticas se van a poder emplear y qué tipo de relaciones entre ellas pueden configurarse para describirlas.

\section{Documentos en contexto}

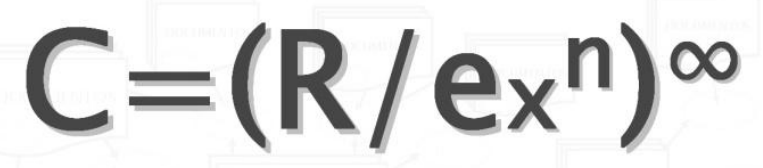

Contexto $=$ Relaciones entre múltiples entidades

Más relaciones $=$ Mejor información

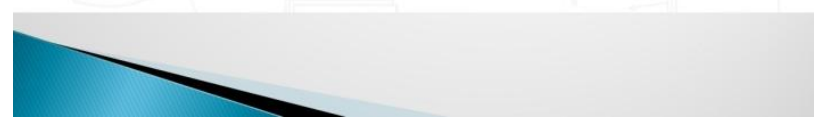

Figura 1: Documentos en contexto 
En este sentido, ninguna de las actuales normas de descripción ofrece una solución para estandarizar esta etapa. Si queremos ofrecer un resultado orientado a cumplir con el objetivo básico de la descripción, que no es otro que hacer accesible y dar a conocer la existencia de documentos representando información, es preciso plantear unas normas nuevas que permitan estandarizar la forma en que se diseñan los sistemas de descripción, con la intención de poder compartir la información que se gestione a través de ellos.

Estas nuevas normas para el diseño de sistemas de descripción deberían resultar de aplicación en cualquier tipo de entorno descriptivo. Por ello, se plantean como un esquema teórico o conceptual que podrá ser implementado de manera flexible y adaptada a las necesidades de cada organización. Además, precisamente por estar concebidas como un modelo conceptual, resultan compatibles con sistemas de descripción analógica tradicional y con sistemas de descripción de documentos digitales.

Para dar respuesta a esta nueva necesidad, ya existen posibles soluciones normativas. La más relevante actualmente es la Norma Española de Descripción Archivística: Modelo Conceptual (NEDA-MC o Modelo Conceptual de Descripción Archivística), cuyo desarrollo comenzó en el año 2007, siendo finalmente aprobado en 2017 por la Comisión de Normas Españolas de Descripción Archivística (CNEDA) como norma para diseñar sistemas de descripción. En NEDA-MC se identifican seis posibles entidades como elementos a describir, así como las posibles relaciones entre dichas entidades. Presta especial atención a las relaciones entre Documentos, Agentes, Funciones y Normas, por considerarse las entidades básicas para definir adecuadamente el contexto documental. 
Pero el contenido regulador del NEDA-MC no se queda limitado a una simple identificación de entidades y relaciones, sino que también ofrece un esquema de los atributos descriptivos, es decir, las propiedades informativas básicas, para describir Documentos, Agentes, Funciones, las relaciones y las propias descripciones que se realicen. Esta relación de atributos permite ejercer como enlace entre los diversos esquemas descriptivos o normas de estructura que, si bien pueden resultar incompatibles de forma directa entre sí, a la hora de analizar la información ofrecida en dichos estándares, son compatibles con el esquema de atributos de la norma española. Esto facilita en última instancia la compatibilidad a través del NEDA-MC de ese conjunto de normas actualmente en vigor, y sobre el cual pendía tanto esa sensación de desencanto como la incompatibilidad (Figura 2).

Además del NEDA-MC, también hay que tener en cuenta la existencia de otro proyecto normativo de ámbito internacional con el mismo objetivo. Se trata del Modelo Conceptual de Descripción Archivística Internacional, conocido como $\mathrm{RiC}-\mathrm{CM}$, por la abreviatura de Records in Contexts: a Conceptual Model for Archival Description, cuyo primer borrador fue difundido en septiembre de 2016. Hoy por hoy se encuentra todavía en fase de análisis de comentarios para poder adoptar formalmente una versión definitiva.

El RiC-CM es el resultado del trabajo realizado entre 2012 y 2016 por el Consejo Internacional de Archivos a través del Grupo de Expertos en Descripción Archivística (ICA/EGAD), y pretende ofrecer una solución al mismo problema planteado anteriormente: la regulación del diseño de sistemas de descripción como paso necesario para estandarizar adecuadamente la descripción archivística. 


\section{Compatibilidad de NEDA-MC}

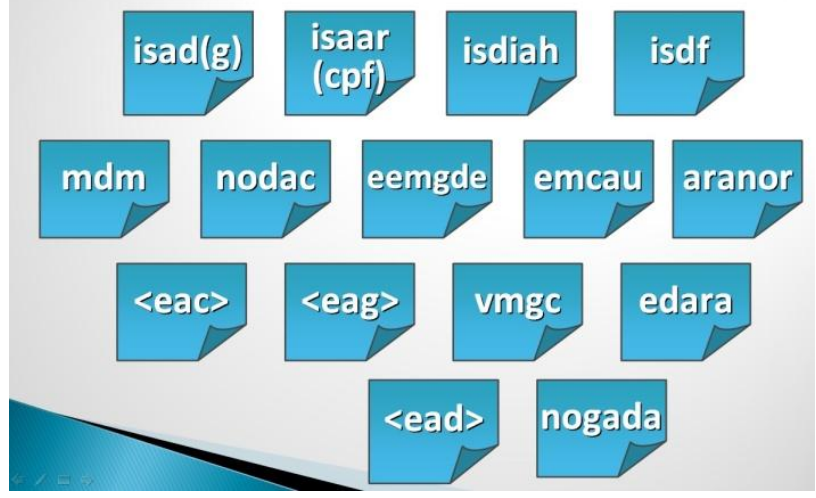

Figura 2: Compatibilidad de NEDA-MC

Si bien es cierto que el NEDA-MC es una norma que ha sido ya aprobada formalmente en 2017 y que el RiC-CM se encuentra todavía en fase de borrador, comparando el contenido de ambas normas puede resultar algo chocante el hecho de que, a pesar de estar planteadas para dar respuesta a una misma necesidad y utilizar un modelado conceptual, el contenido de ambos modelos no sea el mismo. Así, algunas de las diferencias que se pueden identificar a simple vista entre ambos modelos son las siguientes:

- En el NEDA-MC se despliegan 6 entidades frente a las 14 que se plantean en el RiC-CM.

- En el NEDA-MC se identifica unas 40 relaciones entre entidades, frente a las más de 700 del RiC-CM.

- Algunos de los atributos incluidos en el NEDA-MC no parecen coincidir con los que se plantean en el RiC-CM (en cuyo modelo reciben el nombre de properties). 
Esta situación puede inducir a ver inicialmente una incompatibilidad entre ambos modelos, pero si planteamos un estudio comparativo más detallado entre ambas normas, podemos comprobar que no hay tal incompatibilidad.

En primer lugar, hay que tener en cuenta que el RiC-CM se encuentra todavía en fase de borrador, y puede ocurrir que algunas de estas diferencias no resulten tales en la versión definitiva.

En segundo lugar, aunque el NEDA-MC identifica 6 entidades, en algunas de ellas admite la existencia de tipos de entidad, alcanzando un mínimo de 20 posibles tipos, los cuales resultan plenamente compatibles con las entidades identificadas en el RiC-CM (Figura 3).

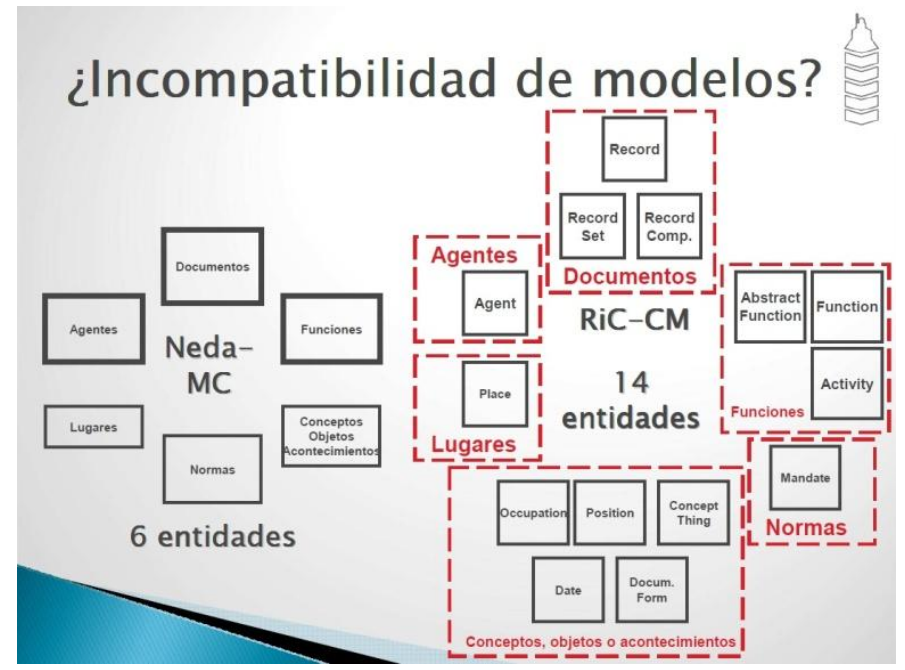

Figura 3: ¿Incompatibilidad de modelos? 
En tercer lugar, el NEDA-MC identifica un número muy limitado de relaciones. Sin embargo, en ningún momento las considera como únicas y exclusivas, sino que admite la posibilidad de ampliar las relaciones entre entidades siempre que se precise, aceptando que las relaciones que quedan identificadas en el NEDA-MC son aquellas que pueden resultar básicas o de uso generalizado.

Por último, los atributos del NEDA-MC son compatibles con las normas internacionales de descripción archivística, así como con las demás normas de descripción actualmente en vigor. Por ello, cuando se adopte formalmente el $\mathrm{RiC}-\mathrm{CM}$ con sus atributos, se podrá plantear su compatibilidad o, si fuera necesario, su posible revisión en una nueva versión.

\section{Conclusiones}

A la vista de que las normas de descripción adoptadas hasta el año 2015 no permitían dar una respuesta adecuada a la nueva necesidad planteada en torno a los requisitos funcionales que deben cumplir los sistemas de descripción, entre los que se incluyen todos aquellos sistemas de gestión orientados a la administración electrónica, la comunidad archivística ha venido tomando cartas en el asunto y, finalmente, gracias a la adopción del NEDA-MC en el ámbito español y a la previsible adopción internacional del RiC-CM, esta carencia se verá solventada.

A partir de ahora, ya existen estándares para diseñar los sistemas donde se gestionan las descripciones archivísticas de documentos, instituciones, personas, funciones y otras muchas entidades de uso. Tan sólo falta que empiecen a aplicarse en el diseño de los futuros sistemas de descripción, o que los sistemas implementados hasta ahora reconduzcan sus 
arquitecturas para cumplir con la finalidad última de la normalización de la descripción: poder compartir la información.

Puesto que la administración viene experimentando un profundo cambio en su propia naturaleza y que, a su vez, dicho cambio conlleva una revisión total del modelo de gestión documental que deriva en una modificación sustancial del escenario descriptivo, cabe concluir que lo más lógico es que las normas de descripción que sirven para regular ese escenario, deben también evolucionar en la misma línea. Es por este motivo que, como queda demostrado a través del NEDA-MC y del RiCCM, ya se está trabajando en unas nuevas normas que se adapten a esta nueva administración, a esos nuevos modelos de gestión documental y a los nuevos escenarios descriptivos.

\section{Bibliografía}

Comisión de Normas Españolas de Descripción Archivística (2017). NEDA-MC: Modelo conceptual de descripción archivística: Entidades, relaciones y atributos. Recuperado de <http://www.mecd.gob.es/cultura/areas/archivos $/ \mathrm{mc} / \mathrm{cneda} /$ documentacion/normas/neda-mc.html $>$.

International Council on Archives. Experts Group on Archival Description. (2016). RiC-CM. Records in Contexts: a conceptual model for archival description. Consultation draft v0.1. Recuperado de <http://www.ica. org/sites/default/files/RiC-CM-0.1.pdf>.

Requejo Zalama, J. (2015). Auge y caída de la descripción multinivel: el nuevo entorno multi-dimensional de la descripción archivística. En Actas de las VIII Jornadas Archivando, Valor, Sociedad y Archivos (pp. 23-39). Recuperado de $\langle$ https://dialnet.unirioja.es/congreso/edicion/8097>.

Requejo Zalama, J. (2016). NEDA: modelos de descripción y modelos de presentación. Tábula, 19, 299-309. Recuperado de <http://publicacio nes.acal.es/index.php/tabula/issue/view/57>. 